Engineering Optimization

Vol. 00, No. 00, Month 200x, 1-10

\title{
SUPPLEMENT
}

\section{A supplement to \\ $A$ tractable approximation of non-convex chance constrained optimization with non-Gaussian uncertainties}

\author{
Abebe Geletu $^{a *}$, Michael Klöppel $^{a}$, Armin Hoffmann $^{b}, \mathrm{Pu} \mathrm{Li}^{a}$ \\ ${ }^{a}$ Department of Simulation and Optimal Processes, Institute for Automation and \\ Systems Engineering, Technische Universität Ilmenau, P.O. Box 1005 65, 98684 \\ Ilmenau, Germany; ${ }^{b}$ Department of Operations Research and Stochastic, Institute \\ of Mathematics, Technische Universität Ilmenau, P.O. Box 1005 65, 98684 \\ Ilmenau, Germany \\ (Received 00 Month 20xx; final version received 00 Month 20xx)
}

\section{Introduction}

This online publication is a supplement to the article $A$ tractable approximation of nonconvex chance constrained optimization with non-Gaussian uncertainties by A. Geletu, M. Klöppel, A. Hoffmann and P. Li. This supplement provides proofs of major theorems that are left out from the main manuscript. To make the reading of this document easier, theorems are restated again here along with their corresponding proofs. Basic mathematical definitions are also provided with some additional references. All other theorems, propositions, assumptions, etc., referenced here are found in the original manuscript.

\section{Proofs propositions and theorems}

PROPOSITION 3.3 Suppose Assumption 3.2 holds true. Then

(1) $\Theta(\tau, u, \cdot)$ is a strictly increasing function w.r.t. $s \in \mathbb{R}$;

(2) $\Theta(\cdot, u, s)$ is a non-decreasing function w.r.t. $\tau$ for $0<\tau<1$;

(3) and

$$
\lim _{\tau \searrow 0^{+}} \Theta(\tau, u, s)=\left\{\begin{array}{l}
1, \text { if } s \geq 0 \\
0, \text { if } s<0
\end{array}\right.
$$

uniformly for $u \in \mathcal{U}$ and uniformly for $s \in(-\infty,-\varepsilon] \cup[0,+\infty)$ and arbitrary $\varepsilon>0$.

*Corresponding author. Email: abebe.geletu@tu-ilmenau.de

ISSN: 0305-215X print/ISSN 1029-0273 online

(C) 200x Taylor \& Francis

DOI: 10.1080/0305215X.YYYY.CATSid

http://www.informaworld.com 
Proof Part (1) follows through an elementary analysis. Since $u$ is fixed, the dependence of $m_{1}$ and $m_{2}$ on $u$ can be dropped for clarity of presentation. Thus, to verify part (2), differentiate $\Theta(\tau, u, s)$ w.r.t. $\tau$ to obtain

$$
\begin{aligned}
\frac{\partial \Theta(\tau, u, s)}{\partial \tau} & =\frac{m_{1}}{\left[1+m_{2} \tau e^{-s / \tau}\right]}+\frac{1+m_{1} \tau}{\left[1+m_{2} \tau e^{-s / \tau}\right]^{2}}\left[-m_{2} e^{-s / \tau}-m_{2}\left(\frac{s}{\tau}\right) e^{-s / \tau}\right] \\
& =\frac{1}{\left[1+m_{2} \tau e^{-s / \tau}\right]^{2}}\left[m_{1}\left(1+m_{2} \tau e^{-s / \tau}\right)-m_{2}\left(1+m_{1} \tau\right)\left(1+\frac{s}{\tau}\right) e^{-s / \tau}\right] .
\end{aligned}
$$

Considering only the factor on the right hand side, it follows that

$$
\begin{aligned}
{\left[m_{1}\left(1+m_{2} \tau e^{-s / \tau}\right)-m_{2}(1\right.} & \left.\left.+m_{1} \tau\right)\left(1+\frac{s}{\tau}\right) e^{-s / \tau}\right] \\
= & m_{1}-m_{2}\left(1+\frac{s}{\tau}\right) e^{-s / \tau}-m_{1} m_{2} s e^{-s / \tau} \\
& =m_{1}-m_{2}\left(1+\frac{s}{\tau}\right) e^{-s / \tau}-m_{1} m_{2} \tau\left(\frac{s}{\tau}\right) e^{-s / \tau}
\end{aligned}
$$

The standard inequality $(1+t) e^{-t} \leq 1$ holds true for any $t \in \mathbb{R}$. Hence, replacing $t$ by $\frac{s}{\tau}$ in the inequality, it follows that

$$
\begin{aligned}
{\left[m_{1}\left(1+m_{2} \tau e^{-s / \tau}\right)-m_{2}(1\right.} & \left.\left.+m_{1} \tau\right)\left(1+\frac{s}{\tau}\right) e^{-s / \tau}\right] \\
& \geq m_{1}-m_{2}-m_{1} m_{2} \tau \\
& >m_{1}-m_{2}\left(1+m_{1}\right) \quad(\text { since } 0<\tau<1) \\
& \geq m_{1}-\frac{m_{1}}{1+m_{1}}\left(1+m_{1}\right)=0 .
\end{aligned}
$$

Consequently, $\frac{\partial \Theta(\tau, u, s)}{\partial \tau}>0$. Therefore, for any value of $s$ (positive or negative) and $u$, the function $\Theta(\tau, u, s)$ is strictly increasing with respect to $\tau$.

The simple estimation

$$
\begin{aligned}
& |\Theta(\tau, u, s)-1| \leq \tau C_{1}, \text { for } s \geq 0 \\
& \Theta(\tau, u, s) \leq \frac{1+C_{1} \tau}{1+C_{3} \tau \exp \left(\frac{\varepsilon}{\tau}\right)}, \text { for } s \leq-\varepsilon
\end{aligned}
$$

yields part (3).

The proof of Theorem 4.2. below needs definitions and properties of convergence of sequences of sets. Hence, given a sequence $\left\{A_{k}\right\}_{k=1}^{+\infty}$ of (closed) sets in $\mathbb{R}^{m}$, basically there are two types of limits (cf. Aubin \& Cellina (1984), Aubin \& Frankwoska (1990), 
Kisielewicz (1991) for details):

$$
\begin{aligned}
& \limsup _{k \rightarrow \infty} A_{k}=\left\{x \in \mathbb{R}^{n} \mid \liminf _{k \rightarrow \infty} \operatorname{dist}\left(x, A_{k}\right)=0\right\}, \\
& \text { and } \\
& \liminf _{k \rightarrow \infty} A_{k}=\left\{x \in \mathbb{R}^{n} \mid \lim _{k \rightarrow \infty} \operatorname{dist}\left(x, A_{k}\right)=0\right\},
\end{aligned}
$$

where $\operatorname{dist}(x, A)=\inf _{z \in A}\|x-z\|$ and $\|\cdot\|$ is the Euclidean norm. If $\limsup \sup _{k \rightarrow \infty} A_{k}=\liminf _{k \rightarrow \infty} A_{k}$, then it is said that the limit of the sequence $\left\{A_{k}\right\}_{k=1}^{+\infty}$ exists and is denoted by $\lim _{k \rightarrow \infty} A_{k}=\limsup _{k \rightarrow \infty} A_{k}=\liminf _{k \rightarrow \infty} A_{k}$.

THEOREM 4.2 The compactness of $\mathcal{U}$, the monotonicity and continuity of $\psi(\cdot, u)$ with respect to $\tau$ as well as properties P1-P3 imply that, for each decreasing zero sequence $\left\{\tau_{k}\right\}_{k \in \mathbb{N}}$ and for a regular chance constraint, the relation

$$
\lim _{k \rightarrow \infty} M\left(\tau_{k}\right)=\mathcal{K}
$$

holds true.

Proof Take an arbitrary decreasing zero sequence $\left\{\tau_{k}\right\}_{k \in \mathbb{N}}$. Using property P3, due to the compactness of $\mathcal{U}$ and continuity of $\psi(\tau, u),\left\{M\left(\tau_{k}\right)\right\}_{k \in \mathbb{N}}$ is a monotonic sequence of compact sets (i.e. $\left.M\left(\tau_{k}\right) \subset M\left(\tau_{k+1}\right)\right)$. It follows that

$$
\liminf _{k \rightarrow \infty} M\left(\tau_{k}\right)=\limsup _{k \rightarrow \infty} M\left(\tau_{k}\right)
$$

Since $\lim \sup _{k \rightarrow \infty} M\left(\tau_{k}\right) \subset \mathcal{K}$ is obvious, it remains to show that $\lim _{\sup } \sup _{k \rightarrow \infty} M\left(\tau_{k}\right) \supset \mathcal{K}$. Suppose $u \in \mathcal{K}$, then there are two cases:

Case 1: $E[h(u, \xi)]<1-\alpha$.

Since $E[h(u, \xi)]=\inf _{\tau \in(0,1)} \psi(\tau, u)$ and $\psi(\cdot, u)$ is non-decreasing and continuous, there is some $k_{0}$ such that $\psi\left(\tau_{k}, u\right)<1-\alpha$, for all $k \geq k_{0}$. Hence, $u \in M\left(\tau_{k}\right)$ for all $k \geq k_{0}$ which yields $u \in \lim \sup M\left(\tau_{k}\right)$.

Case 2: $E[h(u, \xi)]=1-\alpha$.

Using the regularity Assumption 4.1, there is a sequence $\left\{u_{k}\right\}_{k \in \mathbb{N}}$ in $\mathcal{U}$ such that $\lim _{k \rightarrow \infty} u_{k}=u$ and $\operatorname{Pr}\left\{g\left(u_{k}, \xi\right) \leq 0\right\}>\alpha$. This implies that $E\left[h\left(u_{k}, \xi\right)\right]<1-\alpha$. Hence, by property $\mathrm{P} 2$, for each $u_{k}$ there is a sufficiently small $\tau_{k}$ such that $E\left[h\left(u_{k}, \xi\right)\right]<$ $\psi\left(\tau_{k}, u_{k}\right)<1-\alpha$. Hence, $u_{k} \in M\left(\tau_{k}\right)$ for all $k$. This implies that $u \in \lim \sup _{k \rightarrow \infty} M\left(\tau_{k}\right)$.

Observe that, by using property P3 in conjunction with P2, the parameters $\tau_{k}$ can be chosen so that $\left\{\tau_{k}\right\}_{k \in \mathbb{N}}$ is a decreasing zero sequence. Therefore, $\lim _{k \rightarrow \infty} M\left(\tau_{k}\right)=\mathcal{K}$.

COROLLARY 4.3 Let $\left\{\tau_{k}\right\}_{k \in \mathbb{N}} \subset(0,1)$ be any sequence that decreases to zero. Then Assumption 4.1 implies that

$$
\lim _{k \rightarrow \infty} H\left(M\left(\tau_{k}\right), \mathcal{K}\right)=0
$$


Proof Since $\left\{M\left(\tau_{k}\right)\right\}_{k \in \mathbb{N}}$ is a sequence of compact sets, $M\left(\tau_{k}\right) \subset \mathcal{U}, \mathcal{U}$ is a compact set, $\lim _{k \rightarrow \infty} M\left(\tau_{k}\right)=\mathcal{K}$ and $\mathcal{K}$ is a compact set, it follows (see Remark 1.2 of Kisielewicz (1991), also Theorem 5.2.4 of Aubin (1999)) that

$$
\lim _{k \rightarrow \infty} H\left(M\left(\tau_{k}\right), \mathcal{K}\right)=0
$$

THEOREM 4.4 (Approximability of optimal solutions)

(1) Let $\left\{u_{k}\right\}_{k \in \mathbb{N}}$ be a sequence such that $u_{k}$ is a local optimal solution of the $N L P_{k}, k=$ $1,2, \ldots$ Then there is a subsequence $\left\{u_{k_{l}}\right\}_{l \in \mathbb{N}}$ of $\left\{u_{k}\right\}_{k \in \mathbb{N}}$ such that $u_{k_{l}} \rightarrow u^{*}$, there is an open ball $B\left(u^{*}\right)$ around $u^{*}, u^{*} \in \mathcal{K} \cap B\left(u^{*}\right)$ and

$$
E\left[f\left(u^{*}, \xi\right)\right]=\min _{u \in \mathcal{K} \cap B\left(u^{*}\right)} E[f(u, \xi)]
$$

i.e., $u^{*}$ is a local optimal solution of (CCOPT).

(2) Conversely, if $u_{0}$ is a strict local minimizer of CCOPT, then there is a sequence of local minimizers $u_{k}$ of $N L P_{k}$ which converges to $u_{0}$.

Proof

(1) Since $\mathcal{U}$ is a compact set and $\left\{u_{k}\right\}_{k \in \mathbb{N}} \subset \mathcal{U}$, there is a convergent subsequence $\left\{u_{k_{l}}\right\}_{l \in \mathbb{N}}$ such that $u_{k_{l}} \rightarrow u^{*}$. Since $u_{k_{l}} \in M\left(\tau_{k_{l}}\right)$ and (according to Theorem 4.2) $\lim \sup _{k \rightarrow \infty} M\left(\tau_{k}\right)=\mathcal{K}$, it follows that $u^{*} \in \mathcal{K}$. For a sufficiently large $l$, there is a ball $B\left(u^{*}\right)$ such that $u_{k_{l}} \in \mathcal{K} \cap B\left(u^{*}\right)$ are minimizers of $\mathrm{NLP}_{k_{l}}$.

Assume now that $u^{*} \in \mathcal{K} \cap B\left(u^{*}\right)$ is not a local optimum point of CCOPT. This implies that there is $\widetilde{u} \in \mathcal{K} \cap B\left(u^{*}\right)$ such that

$$
E\left[f\left(u^{*}, \xi\right)\right]>E[f(\widetilde{u}, \xi)]
$$

Using Corollary 4.3, there is a sequence $\left\{z_{k}\right\}_{k \in \mathbb{N}}$ such that $z_{k} \in M\left(\tau_{k}\right) \cap B\left(u^{*}\right)$ and $z_{k} \rightarrow \widetilde{u}$. Thus, for a subsequence $\left\{z_{k_{l}}\right\}$ it follows that

$$
\left.E\left[f\left(z_{k_{l}}, \xi\right)\right] \geq E\left[f\left(u_{k_{l}}, \xi\right)\right] \text { (by the local optimality of } u_{k_{l}}\right) \text {. }
$$

This implies that

$$
E[f(\widetilde{u}, \xi)]=\lim _{k_{l} \rightarrow \infty} E\left[f\left(z_{k_{l}}, \xi\right)\right] \geq \lim _{k_{l} \rightarrow \infty} E\left[f\left(u_{k_{l}}, \xi\right)\right]=E\left[f\left(u^{*}, \xi\right)\right]>E[f(\widetilde{u}, \xi)]
$$

But this is a contradiction. Therefore, $u^{*}$ should be a local optimal solution of CCOPT.

(2) Suppose $u_{0}$ be a strict local optimal solution of CCOPT. Let $F(u)>F\left(u_{0}\right)$ for all $u \in \operatorname{cl} B\left(u_{0}\right)$ with $p(u) \geq \alpha$ for a bounded ball $B\left(u_{0}\right)$ around $u_{0}$. Let further that $F(u) \geq F\left(u_{k}\right)$ for all $u \in \operatorname{cl} B\left(u_{0}\right)$ with $1-\psi\left(\tau_{k}, u\right) \geq \alpha$. By the compactness of the closed ball such a $u_{k}$ exists. Without loss of generality, $\lim _{k \rightarrow \infty} u_{k}=: u^{*}$. Since $H\left(M\left(\tau_{k}\right) \cap \operatorname{cl} B\left(u_{0}\right), \mathcal{K} \cap \operatorname{cl} B\left(u_{0}\right)\right) \rightarrow 0$, the continuity of $F$ implies that

$$
F(u) \geq F\left(u^{*}\right) \text { for all } u \in \operatorname{cl} B\left(u_{0}\right) \text { with } p(u) \geq \alpha .
$$


Hence $F\left(u^{*}\right)=F\left(u_{0}\right)$. If $u^{*} \neq u_{0}$, then this leads to a contradiction to the assumption that $u_{0}$ is a strict local minimizer. Consequently, $u_{k}$ converge to $u_{0}$ and there is a $k_{0}$ such for all $k>k_{0}, u_{k}$ belongs to the interior of $B\left(u_{0}\right)$. These $u_{k}$ are all local solutions of $\mathrm{NLP}_{k}$.

THEOREM 4.9 Let $W$ be an open bounded subset of $\mathcal{U}$ and $\tilde{\Omega}$ be an open set with $\Omega \subset \tilde{\Omega}$. Define $B(u, \varepsilon):=\{\xi \in \tilde{\Omega}|| g(u, \xi) \mid<\varepsilon\}$. Then for each $u \in W$ and each $\varepsilon>0$ it follows that

i) $\quad \lim _{k \rightarrow \infty} \sup _{u \in W} \mid \int_{\widetilde{\Omega} \backslash B(u, \varepsilon)}\left[\frac{\partial \Theta\left(\tau_{k}, u, s\right)}{\partial m_{1}} \nabla_{u} m_{1}(u)+\left.\frac{\partial \Theta\left(\tau_{k}, u, s\right)}{\partial m_{2}} \nabla_{u} m_{2}(u)\right|_{s=g(u, \xi)}\right.$ $\left.+\left.\frac{\partial \Theta\left(\tau_{k}, u, s\right)}{\partial s}\right|_{s=g(u, \xi)} \nabla_{u} g(u, \xi)\right] \phi(\xi) d \xi \mid=0$

ii) $\quad \lim _{k \rightarrow \infty} \sup _{u \in W} \mid \int_{B(u, \varepsilon)}\left[\frac{\partial \Theta\left(\tau_{k}, u, s\right)}{\partial m_{1}} \nabla_{u} m_{1}(u)+\left.\frac{\partial \Theta\left(\tau_{k}, u, s\right)}{\partial m_{2}} \nabla_{u} m_{2}(u)\right|_{s=g(u, \xi)} \phi(\xi) d \xi \mid=0\right.$

iii) $\lim _{k \rightarrow \infty} \nabla \psi\left(\tau_{k}, u\right)=\lim _{k \rightarrow \infty} \int_{B(u, \varepsilon)}\left[\left.\frac{\partial \Theta\left(\tau_{k}, u, s\right)}{\partial s}\right|_{s=g(u, \xi)} \nabla_{u} g(u, \xi)\right] \phi(\xi) d \xi$ uniformly on $W$.

Proof A detailed proof of this theorem takes several pages. Thus, this section provides only a sketch of the idea of the proof.

- Referring to Proposition 3.3 it should be noted that the function $\lim _{\tau \searrow 0^{+}} \Theta(\tau, u, s)=\left\{\begin{array}{l}1, \text { if } s \geq \varepsilon, \\ 0, \text { if } s<-\varepsilon,\end{array}\right.$ for an $\varepsilon>0$. Consequently, for $|s|>\varepsilon$ the limit of the derivative $\lim _{\tau} \searrow^{+} \nabla_{u} \Theta(\tau, u, s)=0$. Thus, the main contribution to the derivative comes when $-\varepsilon<s<\varepsilon$. Thus, replacing $s=g(u, \xi)$ gives an idea on how the proof should proceed.

- Let $u_{0} \in W$ and let

$$
\Gamma_{0}\left(u_{0}\right)=\left\{\xi \in \widetilde{\Omega} \mid g\left(u_{0}, \xi\right)=0\right\} .
$$

Assume that $\Gamma_{0}\left(u_{0}\right) \neq \emptyset$ (for $\Gamma_{0}\left(u_{0}\right)=\emptyset$ the discussion below follows trivially). Hence, there is $\tilde{\xi} \in \widetilde{\Omega}$ such that $\left(u_{0}, \tilde{\xi}\right) \in \Gamma_{0}\left(u_{0}\right)$. Furthermore, define the set

$$
\Gamma_{g}=\{(u, \xi, s) \mid s=0, g(u, \xi)=0,(u, \xi) \in W \times \widetilde{\Omega}\} .
$$

Since $\Gamma_{0}\left(u_{0}\right) \neq \emptyset$, it follows that $\Gamma_{g} \neq \emptyset$.

- The set $\Gamma_{g}$ is a compact set. For each $(\tilde{u}, \tilde{\xi}, \tilde{s}) \in \Gamma_{g}$ it follows that $g(\tilde{u}, \tilde{\xi})=0$ and $\tilde{s}=0$. According to Assumption 4.5, $\nabla_{\xi} g(\tilde{u}, \tilde{\xi}) \neq 0$. This implies there is a component $\xi_{\beta}$ of $\xi=\left(\xi_{1}, \xi_{2}, \ldots, \xi_{p}\right)$ such that $\frac{\partial}{\partial \xi_{\beta}} g(\tilde{u}, \tilde{\xi}) \neq 0$. Without loss of generality, let $\xi_{\beta}=\xi_{p}$ and define $\eta=\left(\xi_{1}, \xi_{2}, \ldots, \xi_{p-1}\right)$. Hence, 
(a) according to the Implicit Function and Open Mapping Theorems, there are open neighborhoods $\widetilde{U}, \widetilde{V}$ and $(-\varepsilon, \varepsilon)$ of $\widetilde{u}, \tilde{\eta}$ and $\tilde{s}=0$, respectively; an interval $(a, b)$ with $a<\tilde{\xi}_{\beta}<b$ and a unique surjective mapping $q$

$$
q: \tilde{U} \times \tilde{V} \times(-\varepsilon, \varepsilon) \rightarrow(a, b)
$$

such that $q(\tilde{u}, \tilde{\eta}, \tilde{s})=q(\tilde{u}, \tilde{\eta}, 0)=\widetilde{\xi}_{\beta}$ and $g\left(u,(\eta, \underbrace{q(u, \xi, s)}_{=\xi_{\beta}})\right)=s$, for each $(u, \eta, s) \in$ $\tilde{U} \times \tilde{V} \times\left(-s_{0}, s_{0}\right)$.

(b) The family of sets $\left\{(\tilde{U} \times(\tilde{V} \times(a, b)) \times(-\varepsilon, \varepsilon)) \mid(u, \xi, 0) \in \Gamma_{g}\right\}$ defines an open covering of the compact set $\Gamma_{g}$. Hence, there is a finite collection $\left.\left\{\left(\tilde{U}_{i} \times\left(\tilde{V}_{i} \times\left(a_{i}, b_{i}\right)\right) \times\left(-\varepsilon_{i}, \varepsilon_{i}\right)\right)\right) \mid i=1, \ldots, z\right\}$ that covers $\Gamma_{g}$ with a corresponding set of surjective mappings $\left\{q_{i} \mid i=1, \ldots, z\right\}$. Define now that

$$
\varepsilon_{0}:=\min \left\{\bar{\varepsilon}, \varepsilon_{1}, \ldots, \varepsilon_{z}\right\}
$$

Let $s=g(u, \xi)$. Then, for each $u \in \bigcup_{i=1}^{z} \tilde{U}_{i}$

$$
B\left(u, \varepsilon_{0}\right) \subset \bigcup_{i=1}^{z}\left(\widetilde{V}_{i} \times\left(a_{i}, b_{i}\right)\right)
$$

with $B\left(u, \varepsilon_{0}\right)=\left\{\xi \mid-\varepsilon_{0}<g(u, \xi)<\varepsilon_{0}\right\}$. Figure 1 indicates that the set $\Gamma_{0}\left(u_{0}\right)$ is contained in the open set $B\left(u, \varepsilon_{0}\right)$.

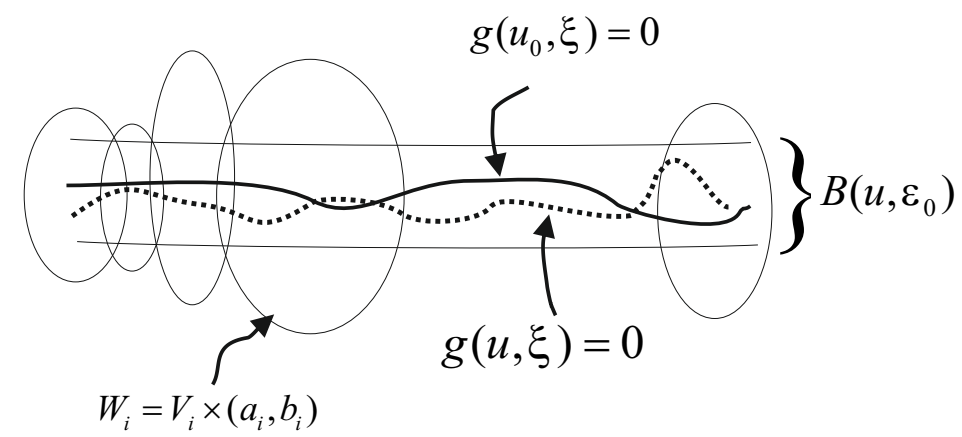

Figure 1. An $\varepsilon$-tube around $\Gamma_{0}\left(u_{0}\right)$

(c) Let $M:=\cup_{i=1}^{z}\left(\tilde{U}_{i} \times\left(\tilde{V}_{i} \times\left(a_{i}, b_{i}\right)\right)\right)$ and the family $\varphi_{i} \in C^{\infty}(M, \mathbb{R}), i=1, \ldots, z$, be a partition of unity of the system $\left\{\tilde{U}_{i} \times\left(\tilde{V}_{i} \times\left(a_{i}, b_{i}\right)\right) \mid i=1, \ldots, z\right\}$; i.e., $\sum_{i=1}^{z} \varphi_{i}(u, \xi)=1$ on $M, 0 \leq \varphi_{i}(u, \xi) \leq 1, i=1, \ldots, z$, and compact support $\operatorname{supp} \varphi_{i} \subset \tilde{U}_{i} \times\left(\tilde{V}_{i} \times\left(a_{i}, b_{i}\right)\right), i=1, \ldots, z$. 
- Hence, it follows that

$$
\begin{aligned}
\left.\int_{B\left(u, \varepsilon_{0}\right)} \nabla_{u} \Theta\left(\tau_{k}, u, s\right)\right|_{s=g(u, \xi)} \phi(\xi) d \xi & \\
= & \left.\sum_{i=1}^{z} \int_{V_{i} \times\left(a_{i}, b_{i}\right),|g(u, \xi)|<\varepsilon_{0}} \nabla_{u} \Theta\left(\tau_{k}, u, s\right)\right]_{s=g(u, \xi)} \phi(\xi) \varphi_{i}(u, \xi) d \xi .
\end{aligned}
$$

Furthermore, use the coordinate transformation

$$
\xi_{j}=\left\{\begin{array}{cc}
\eta_{j} & \text { if } j \neq \beta \\
q(u, \eta, s) & \text { if } j=\beta
\end{array}, \quad j=1,2, \ldots, p\right.
$$

to write

$$
\begin{aligned}
&\left.\sum_{i=1}^{z} \int_{V_{i} \times\left(a_{i}, b_{i}\right),|g(u, \xi)|<\varepsilon_{0}} \nabla_{u} \Theta\left(\tau_{k}, u, s\right)\right|_{s=g(u, \xi)} \phi(\xi) \varphi_{i}(u, \xi) d \xi \\
&=\sum_{i=1}^{z} \int_{V_{i} \times\left(a_{i}, b_{i}\right),|g(u, \xi)|<\varepsilon_{0}} Q\left(\tau_{k}, u, \xi, g(u, \xi)\right) \mu_{i}(u, \xi) d \xi
\end{aligned}
$$

where

$$
\begin{array}{r}
Q\left(\tau_{k}, u, \xi, g(u, \xi)\right):=\left[\frac{\partial \Theta\left(\tau_{k}, u, s\right)}{\partial m_{1}} \nabla_{u} m_{1}(u)+\frac{\partial \Theta\left(\tau_{k}, u, s\right)}{\partial m_{2}} \nabla_{u} m_{2}(u)\right. \\
\left.+\frac{\partial \Theta\left(\tau_{k}, u, s\right)}{\partial s} \nabla_{u} g(u, \xi)\right]_{s=g(u, \xi)}
\end{array}
$$

and $\mu_{i}(u, \xi):=\phi(\xi) \varphi_{i}(u, \xi)$ so that

$$
\begin{gathered}
\sum_{i=1}^{z} \int_{V_{i} \times\left(a_{i}, b_{i}\right),|g(u, \xi)|<\varepsilon_{0}} Q\left(\tau_{k}, u, \xi, g(u, \xi)\right) \phi(\xi) \varphi_{i}(u, \xi) d \xi \\
=\sum_{i=1}^{z} \int_{V_{i}} \int_{-\varepsilon_{i}}^{\varepsilon_{i}} Q\left(\tau_{k}, u,\left(\eta, q_{i}(u, \eta, s)\right)\right) \mu_{i}\left(u, \eta, q_{i}(u, \eta, s)\right) d s d \eta \\
\quad-\sum_{i=1}^{z} \int_{V_{i}} \int_{\varepsilon_{0}}^{\varepsilon_{i}} Q\left(\tau_{k}, u,\left(\eta, q_{i}(u, \eta, s)\right)\right) \mu_{i}\left(u, \eta, q_{i}(u, \eta, s)\right) d s d \eta \\
-\sum_{i=1}^{z} \int_{V_{i}} \int_{-\varepsilon_{i}}^{-\varepsilon_{0}} Q\left(\tau_{k}, u,\left(\eta, q_{i}(u, \eta, s)\right)\right) \mu_{i}\left(u, \eta, q_{i}(u, \eta, s)\right) d s d \eta
\end{gathered}
$$

The rest of the proof uses a sequence of technical Lemmata for elementary properties (which hold uniformly with respect $u \in W$ ) of the expressions $\frac{\partial \Theta(\tau, u, s)}{\partial m_{1}}, \frac{\partial \Theta(\tau, u, s)}{\partial m_{2}}, \frac{\partial \Theta(\tau, u, s)}{\partial s}$, their integrals w.r.t. $s$ and $\nabla_{u} g(u, \xi)$ corresponding to $|s| \geq \varepsilon_{0},|s|<\varepsilon_{0}$ and $\tau_{k} \searrow 0^{+}$, respectively, as well as the properties of the coordinate transformation. Based on the 
Mean-Value Theorem, the inner integration can be removed and thus the delta distributional term $\frac{\partial \Theta(\tau, u, s)}{\partial s}$ remains. Hereby the continuity of the density $\phi(\xi)$ is required.

THEOREM 4.13 Let $\left\{\tau_{k}\right\}_{k \in \mathbb{N}} \subset(0,1)$ be any sequence with $\tau_{k} \searrow 0^{+}$and let Assumptions 4.11 and 4.12 be satisfied. Then the following hold true

(1) If Assumption 4.11 (MFCQ) holds, then

(a) $\lim \sup _{k \rightarrow \infty} \Lambda_{k} \subset \Pi$ and

(b) $\lim \sup _{k \rightarrow \infty} \Lambda_{k}^{0} \subset \Pi_{0}$.

(2) If Assumption 4.12 (LICQ) holds, then $\Pi_{1} \subset \lim \sup _{k \rightarrow \infty} \Lambda_{k}^{0}$.

(1) Take any sequence $\left\{\tau_{k}\right\}_{k \in \mathbb{N}} \subset(0,1)$ such that $\tau_{k} \searrow 0^{+}$. Let $\left(u_{k}, \eta_{k}\right) \in \Lambda_{k}$ such that $\left(u_{k}, \eta_{k}\right) \longrightarrow\left(u_{0}, \eta_{0}\right)$. For each $j \in\{1, \ldots, q\}$ using the estimation

$$
\left\|\nabla \psi_{k, j}\left(u_{k}\right)+\nabla p\left(u_{0}\right)\right\| \leq\left\|\nabla \psi_{k, j}\left(u_{k}\right)+\nabla p_{j}\left(u_{k}\right)\right\|+\left\|-\nabla p_{j}\left(u_{k}\right)+\nabla p_{j}\left(u_{0}\right)\right\|
$$

and applying Proposition 4.9 and the continuity of $\nabla p_{j}(\cdot)$ it follows that

$$
\lim _{k \rightarrow \infty}\left\|\nabla \psi_{k, j}\left(u_{k}\right)+\nabla p\left(u_{k}\right)\right\| \leq \lim _{k \rightarrow \infty} \sup _{u \in \mathcal{U}}\left\|\nabla \psi_{k, j}(u)+\nabla p_{j}(u)\right\|=0
$$

and $\lim _{k \rightarrow \infty}\left\|-\nabla p\left(u_{k}\right)+\nabla p\left(u_{0}\right)\right\|=0$. Consequently,

$$
\sum_{j=1}^{q} \eta_{k, j} \nabla \psi_{k, j}\left(u_{k}\right) \underset{k \rightarrow \infty}{\longrightarrow}-\sum_{j=1}^{q} \eta_{0, j} \nabla p_{j}\left(u_{k}\right)
$$

where $\eta_{j, k}$ and $\eta_{j, 0}$ are the $j$-th components of $\eta_{k}$ and $\eta_{0}$, respectively. Furthermore, the continuous differentiability of $F(u)$ yields that

$$
\nabla F\left(u_{k}\right)+\sum_{j=1}^{q} \eta_{k, j} \nabla \psi_{k, j}\left(u_{k}\right)=0 \quad \underset{k \rightarrow \infty}{\longrightarrow} \nabla F\left(u_{0}\right)-\sum_{j=1}^{q} \eta_{0, j} \nabla p_{j}\left(u_{0}\right)=0 .
$$

The complementarity and non-negativity conditions in $\Pi$ are also consequences of the continuity and non-negativity properties of $p_{j}(u)$ and $\psi_{k, j}(u)$. Hence, it follows that $\left(x_{0}, \eta_{0}\right) \in \Pi$ and

$$
\limsup _{k \rightarrow \infty} \Lambda_{k} \subset \Pi
$$

The proof for $\limsup _{k \rightarrow \infty} \Lambda_{k}^{0} \subset \Pi_{0}$ is the same as in part (1a).

(2) It remains to prove that $\Pi_{1} \subset \lim \sup _{k \rightarrow \infty} \Lambda_{k}^{0}$. Suppose now that $\left(u_{0}, \eta_{0}\right) \in \Pi_{1}$. Since $u_{0}$ is a strict local optimal solution of CCOPT, Theorem 4.4(2) implies that there is sequence $\left\{u_{k}\right\}_{k \in \mathbb{N}}$ such that $u_{k}$ is a local optimal solution of $N L P_{k}$ and $\lim _{k \rightarrow \infty} u_{k}=u_{0}$.

For each $k \in\{1,2,3, \ldots\}$, the local optimality of $u_{k}$ to $\mathrm{NLP}_{k}$ yields that there is $\eta_{k} \in \mathbb{R}_{+}^{q}$ such that $\left(u_{k}, \eta_{k}\right) \in \Lambda_{k}^{0}$. 
Claim: There is a subsequence $\left\{\eta_{k_{l}}\right\}$ of $\left\{\eta_{k}\right\}$ such that

$$
\eta_{k_{l}} \longrightarrow \eta_{0}
$$

Now, from the local optimality of $u_{0}$ and $u_{k}$, it follows that

$$
\left\|\nabla F\left(u_{k}\right)+\sum_{j=1}^{q} \eta_{k, j} \nabla \psi_{k, j}\left(u_{k}\right)-\nabla F\left(u_{0}\right)+\sum_{j=1}^{q} \eta_{0, j} \nabla p_{j}\left(u_{0}\right)\right\| \leq \varepsilon, k=1,2, \ldots
$$

for an arbitrary $\varepsilon>0$. This implies,

$$
\begin{gathered}
\| \nabla F\left(u_{k}\right)+\sum_{j=1}^{q}\left(\eta_{k, j}-\eta_{0, j}\right) \nabla \psi_{k, j}\left(u_{k}\right)-\nabla F\left(u_{0}\right) \\
+\sum_{j=1}^{q} \eta_{0, j}\left(\nabla p_{j}\left(u_{0}\right)+\nabla \psi_{j}\left(\tau_{k}, u_{k}\right)\right) \| \leq \varepsilon, \\
k=1,2, \ldots
\end{gathered}
$$

This yields that

$$
\begin{gathered}
\left\|\sum_{j=1}^{q}\left(\eta_{k, j}-\eta_{0, j}\right) \nabla \psi_{k, j}\left(u_{k}\right)\right\| \leq \varepsilon+\left\|\nabla F\left(u_{k}\right)-\nabla F\left(u_{0}\right)\right\| \\
+\left\|\sum_{j=1}^{q} \eta_{0, j}\left(\nabla p_{j}\left(u_{0}\right)+\nabla \psi_{k, j}\left(u_{k}\right)\right)\right\|, \\
k=1,2, \ldots
\end{gathered}
$$

Consequently,

$$
\begin{gathered}
\left\|\eta_{k}-\eta_{0}\right\|\left\|\sum_{j=1}^{q} \frac{\left(\eta_{k, j}-\eta_{0, j}\right)}{\left\|\eta_{k}-\eta_{0}\right\|+1} \nabla \psi_{k, j}\left(u_{k}\right)\right\| \leq \\
\varepsilon+\left\|\nabla F\left(u_{k}\right)-\nabla F\left(u_{0}\right)\right\|+\left\|\sum_{j=1}^{q} \eta_{0, j}\left(\nabla p_{j}\left(u_{0}\right)+\nabla \psi_{k, j}\left(u_{k}\right)\right)\right\| \\
k=1,2, \ldots
\end{gathered}
$$

Let $\delta_{k . j}:=\frac{\left(\eta_{k, j}-\eta_{0, j}\right)}{\left\|\eta_{k}-\eta_{0}\right\|+1}, j=1,2, \ldots, q$. Then the sequence $\left\{\delta_{k, j}\right\}$, for each $j \in$ $\{1, \ldots, q\}$, is bounded. Hence, there is a subsequence $\left\{\delta_{k_{l}, j}\right\}$ which is convergent and $\delta_{k_{l}, j} \longrightarrow \delta_{0, j}$ for some $\delta_{0, j} \in \mathbb{R}$. Since, $\left\{\psi_{k, j}\left(u_{k}\right)\right\}$ is a convergent sequence, the sequence $\left\{\delta_{k_{l}, j} \psi_{k_{l}, j}\left(u_{k}\right)\right\}$ is convergent and $\delta_{k_{l}, j} \psi_{k_{l}, j}\left(u_{k}\right) \longrightarrow \delta_{0, j} \nabla p_{j}\left(u_{0}\right)$. As 
a result it follows that

$$
\left\|\sum_{j=1}^{q} \frac{\left(\eta_{k_{l}, j}-\eta_{0, j}\right)}{\left\|\eta_{k_{l}}-\eta_{0}\right\|+1} \nabla \psi_{k_{l}, j}\left(u_{k}\right)\right\| \longrightarrow\left\|\sum_{j=1}^{q} \delta_{0, j} \nabla p_{j}\left(u_{0}\right)\right\| .
$$

To show that $\left\|\sum_{j=1}^{q} \delta_{0, j} \nabla p_{j}\left(u_{0}\right)\right\|=0$ only if $\delta_{0, j}=0$ for each $j \in\{1, \ldots, q\}$. Assume that there is $j^{\prime} \in\{1, \ldots, q\}$ such that $\delta_{0, j^{\prime}} \neq 0$ and $\left\|\sum_{j=1}^{q} \delta_{0, j} \nabla p_{j}\left(u^{*}\right)\right\|=$ 0 . This implies $\sum_{j=1}^{q} \delta_{0, j} \nabla p_{j}\left(u^{*}\right)=0$ violating the Assumption 4.12 (LICQ). Consequently,

(i) either $\delta_{0, j}=0$ for each $j \in\{1, \ldots, q\}$;

(ii) or $\left\|\sum_{j=1}^{q} \delta_{0, j} \nabla p_{j}\left(u^{*}\right)\right\| \neq 0$.

The latter case yields

$$
\left\|\eta_{k_{l}}-\eta_{0}\right\| \leq \frac{\varepsilon+\left\|\nabla F\left(u_{k_{l}}\right)-\nabla F\left(u_{0}\right)\right\|+\left\|\sum_{j=1}^{q} \eta_{0, j}\left(\nabla p_{j}\left(u_{0}\right)+\nabla \psi_{k_{l}, j}\left(u_{k_{l}}\right)\right)\right\|}{\left\|\sum_{j=1}^{q} \delta_{k, l} \nabla \psi_{k_{l}, j}\left(u_{k_{l}}\right)\right\|} .
$$

Consequently, both cases (i) and (ii) imply that $\left\|\eta_{k_{l}}-\eta_{0}\right\| \longrightarrow 0$ which verifies the claim and concludes the proof.

\section{Additional References}

\section{References}

Aubin, J.-P. 1999. Mutational and morphological analysis. Boston: Birkhäuser.

Aubin, J.-P., Cellina, A. 1984. Differential Inclusions. Berlin: Springer Verlag. Aubin, J.-P., Frankowska, H. 1990. Set-Valued Analysis. Basel: Birkhäuser.

Kisielewicz, M. 1991. Differential inclusions and optimal control. Warszawa: Polish Scientific Publishers.

Rudin, W. 1976. Principles of mathematical analysis, 3rd ed. New York: McGraw-Hill.

Ruszczyńiski, A., Shapiro, A. 2003. "Optimality and duality in stochatic programs." In Stochastic Programming, Handbooks in Operations Research and Management Science,V. 10, edited by A. Ruszczyniski and A. Shapiro. 65-138. Amsterdam: Elsevier Science. 